\title{
AN INTEGRODIFFERENTIAL EQUATION ASYMPTOTICALLY OF CONVOLUTION TYPE
}

\author{
KENNETH B. HANNSGEN
}

ABSTRACT. The resolvent formula is used to study the asymptotic behavior $(t \rightarrow \infty)$ of solution to integrodifferential equations which are close in some sense to equations of convolution type with integrable resolvents.

I. Introduction. For the problem

$$
x^{\prime}(t)+\int_{0}^{t} b(t, s) x(s) d s=f(t)
$$

$\left({ }^{\prime}=d / d t, \quad t \in \mathbf{R}^{+} \equiv[0, \infty)\right)$ with initial condition $x(0)=x_{0}$, we give conditions on $b$ which ensure that $x \in L^{p}\left(\mathbf{R}^{+}\right)$if $f \in L^{p}\left(\mathbf{R}^{+}\right)$, for some $p \geqslant 1$. We shall assume that, for large $t$ and $s, b(t, s)$ is close to a kernel $a(t-s)$ of convolution type with resolvent $r$ in $L^{1}\left(\mathbf{R}^{+}\right)$. We shall also present some results for related almost linear problems.

Throughout this paper, $\|\varphi\|$ and $\|\varphi\|_{p}$ denote respectively the $L^{1}$ and $L^{p}$ norms of the function $\varphi: \mathbf{R}^{+} \rightarrow \mathbf{R}$. A solution of (1.1) is a locally absolutely continuous function $x: \mathbf{R}^{+} \rightarrow \mathbf{R}$ such that (1.1) holds almost everywhere.

If $b(t, s)=a(t-s) \quad(0<s<t)$ with $a$ locally $L^{1}$ on $\mathbf{R}^{+}$(" $a \in$ $L L^{1}\left(\mathbf{R}^{+}\right)$"), then, for $f \in L L^{1}\left(\mathbf{R}^{+}\right)$,

$$
x(t)=x_{0} r(t)+\int_{0}^{t} r(t-s) f(s) d s \quad(0 \leqslant t<\infty),
$$

where $r$, the (differential) resolvent of $a$, is the solution of

$$
r^{\prime}(t)+\int_{0}^{t} a(t-s) r(s) d s=0, \quad r(0)=1 .
$$

(See [1], for example.) Thus $x \in L^{p}\left(\mathbf{R}^{+}\right)$for all $f \in L^{p}\left(\mathbf{R}^{+}\right)(1 \leqslant p \leqslant \infty)$ if

$$
r \in L^{1}\left(\mathbf{R}^{+}\right) \cap L^{\infty}\left(\mathbf{R}^{+}\right) .
$$

Assuming (1.4), we employ (1.2) and some simple estimates to derive our results for (1.1) with the more general kernel $b(t, s)$. Among previous studies of stability theory for integrodifferential equations, involving the resolvent formula, we mention those of S. I. Grossman and R. K. Miller [1], [2] and of Miller [7].

The author thanks G. S. Jordan for an important correction.

Received by the editors May 30, 1978.

AMS (MOS) subject classifications (1970). Primary 45M05.

(c) 1979 American Mathematical Society 0002-9939/79/0000-0163/\$03.00 
II. Linear equations. Our first result displays the method in its simplest form.

TheOREM 2.1. Let $1 \leqslant p \leqslant \infty$. Let $b \in L L^{1}(S)$, where $S=\{0 \leqslant s \leqslant t<$ $\infty\}$, and suppose there exists $a \in L L^{1}\left(\mathbf{R}^{+}\right)$, with resolvent $r$ satisfying (1.4), such that for each $\varepsilon>0$ there exist $T>0$ and $c \in L^{1}\left(\mathbf{R}^{+}\right)$with $\|c\|<\varepsilon$ and

$$
\begin{gathered}
|b(t+T, s+T)-a(t-s)| \leqslant c(t-s) \text { a.e. in } S, \\
\int_{0}^{\infty}\left[\int_{0}^{T}|b(t+T, s)| d s\right]^{p} d t<\infty .
\end{gathered}
$$

Let $f \in L^{p}\left(\mathbf{R}^{+}\right)$, and let $x$ be a solution of (1.1). Then $x \in L^{p}\left(\mathbf{R}^{+}\right)$.

We discuss and illustrate our results in $\S \mathrm{V}$; for example, we show that the hypotheses of Theorem 2.1 hold with $p=1$ for a large class of $a \in L^{1}\left(\mathbf{R}^{+}\right)$ with $b(t, s)=\alpha(t) \beta(s) a(t-s)$, where $\alpha(t) \rightarrow 1, \beta(t) \rightarrow 1$ as $t \rightarrow \infty$. On the other hand, with $f \equiv 0, b(t, s)=a(t-s)+\beta(s) A(t-s)(\beta(s)=0$ for $s>$ 1) it can happen that $x(\infty)=0$ but $x \notin L^{1}\left(\mathbf{R}^{+}\right)$. The following positive result holds, however.

THEOREM 2.2. Let $x$ be a solution of (1.1) with

$$
b(t, s)=\alpha(t) \beta(s) A(t-s)+\gamma(s) a(t-s),
$$

where

$$
\begin{gathered}
a \in L L^{1}\left(\mathbf{R}^{+}\right) \text {with resolvent } r \in L^{1}\left(\mathbf{R}^{+}\right), r^{\prime} \in L^{1}\left(\mathbf{R}^{+}\right), \\
0 \leqslant A \in L L^{1}\left(\mathbf{R}^{+}\right), \quad \alpha(t) \downarrow 0 \quad(t \uparrow \infty), \quad \alpha A \in L^{1}\left(\mathbf{R}^{+}\right), \\
\beta, \gamma \in L^{\infty}\left(\mathbf{R}^{+}\right), \quad \gamma(t) \rightarrow 1 \quad(t \rightarrow \infty), \\
f \in L^{1}\left(\mathbf{R}^{+}\right) .
\end{gathered}
$$

Then $x \in L^{1}\left(\mathbf{R}^{+}\right)$.

Well-known sufficient conditions for (1.7) are discussed in $\S \mathrm{V}$.

III. Almost linear equations. The results of §II, together with a perturbation theorem of S. I. Grossman and R. K. Miller [1, Theorem 4], immediately yield an existence-stability result for the almost linear equation

$$
x^{\prime}(t)+\int_{0}^{t} b(t, s) x(s) d s+(h x)(t)=f(t), \quad x(0)=x_{0},
$$

where $h: L^{p} \rightarrow L^{p}$ is of higher order with respect to $L^{p}$. (Higher order means $h 0=0$ and $\left\|h \varphi_{1}-h \varphi_{2}\right\|_{p}=o\left\|\varphi_{1}-\varphi_{2}\right\|_{p}$ as $\left\|\varphi_{1}\right\|_{p},\left\|\varphi_{2}\right\|_{p} \rightarrow 0$. Solution is defined as for (1.1).)

COROllary 3.1. Let $b$ satisfy the conditions of Theorem 2.1 [Theorem 2.2], and let $h$ be of higher order with respect to $L^{p}\left(\mathbf{R}^{+}\right)\left[L^{1}\left(\mathbf{R}^{+}\right)\right]$. Then for each $\varepsilon>0$, there exists a number $\eta>0$ such that if $\left|x_{0}\right| \leqslant \eta$ and $\|f\|_{p} \leqslant \eta$ $[\|f\| \leqslant \eta]$, then (3.1) has a unique solution in $L^{p}\left(\mathbf{R}^{+}\right)\left[L^{1}\left(\mathbf{R}^{+}\right)\right]$with $\|x\|_{p} \leqslant \varepsilon$ $\left[\|x\|_{1} \leqslant \varepsilon\right]$. 
Corollary 3.1 holds, of course, for the equation

$$
x^{\prime}(t)+\int_{0}^{t} b(t, s)[x(s)+g(x(s))] d s=f(t)
$$

for suitable $b, g$. Using the method of $\S I$, we can establish a related result; instead of requiring $x_{0}$ and $\|f\|$ to be small, we assume $a$ priori that

$$
x(t) \rightarrow 0 \text { as } t \rightarrow \infty \text {. }
$$

Known sufficient conditions for (3.3), involving the signs of $b$ and its partial derivatives, are discussed in $\S \mathrm{V}$ below.

THEOREM 3.2. Let $b$ and $f$ satisfy the hypotheses of Theorem $2.1(p=1)$ with $a \in L^{1}\left(\mathbf{R}^{+}\right)$or the hypotheses of Theorem 2.2. Let $g \in C(\mathbf{R})$ with $g(x)=o(x)$ $(x \rightarrow 0)$. Suppose $x$ is a solution of (3.2), and assume (3.3). Then $x \in L^{1}\left(\mathbf{R}^{+}\right)$.

IV. Proofs. For Theorem 2.1, let $0<\varepsilon<1 / 2\|r\|$ and choose corresponding $T$ and $c$. Set $y(t)=x(t+T), F(t)=f(t+T)(t>0)$ and make a change of variables in (1.1) to obtain

$$
\begin{aligned}
y^{\prime}(t)+\int_{0}^{t} a(t-s) y(s) d s & \\
& =\int_{0}^{t}[a(t-s)-b(T+t, T+s)] y(s) d s+F_{1}(t)
\end{aligned}
$$

with $y(0)=x(T)$, where

$$
\left|F_{1}(t)\right| \leqslant\left(\max _{0<\tau<T}|x(\tau)|\right) \int_{0}^{T}|b(t+T, s)| d s+|F(t)|,
$$

so that $F_{1} \in L^{p}\left(\mathbf{R}^{+}\right)$. By (1.2), $y=\varphi+\mathcal{L} y$, where

$$
\begin{aligned}
\varphi(t) & =r(t) x(T)+\int_{0}^{t} r(t-\tau) F_{1}(\tau) d \tau \in L^{p}\left(\mathbf{R}^{+}\right), \\
\mathcal{L} y(t) & =\int_{0}^{t} r(t-\tau) \int_{0}^{\tau}[a(\tau-s)-b(\tau+T, s+T)] y(s) d s d \tau,
\end{aligned}
$$

so that $\mathcal{L}: L^{p}\left(\mathbf{R}^{+}\right) \rightarrow L^{p}\left(\mathbf{R}^{+}\right)$satisfies

$$
\|\mathcal{L} z\|_{p} \leqslant\|r\|\|c\|\|z\|_{p} \leqslant \frac{1}{2}\|z\|_{p}
$$

by (1.5). For $0<\rho<\infty$, let

$$
y_{\rho}(t)=y(t) \quad(0 \leqslant t \leqslant \rho), \quad y_{\rho}(t)=0 \quad(\rho<t<\infty) .
$$

Clearly $y_{\rho} \in L^{p}\left(\mathbf{R}^{+}\right)$and

$$
\left|y_{\rho}(t)\right| \leqslant|\varphi(t)|+\left|\mathcal{L} y_{\rho}(t)\right| \quad(0 \leqslant t<\infty) .
$$

By Minkowski’s inequality and (4.2),

$$
\left\|y_{\rho}\right\|_{p} \leqslant 2\|\varphi\|_{p} \quad(0<\rho<\infty) .
$$

It follows that $y \in L^{p}\left(\mathbf{R}^{+}\right)$as claimed. 
For Theorem 2.2, choose $T$ so large that

$$
\left\|r^{\prime}\right\|\|1-\gamma(T+\cdot)\|_{\infty}+\|r\|\|A(\cdot) \alpha(T+\cdot)\|\|\beta\|_{\infty}<\frac{1}{2} .
$$

$T$ exists, since $\gamma \rightarrow 1$ and for $M, N>0$

$$
\int_{0}^{\infty} A(t) \alpha(M+N+t) d t \leqslant \alpha(M+N) \int_{0}^{M} A(t) d t+\int_{M}^{\infty} A(t) \alpha(t) d t
$$

we obtain (4.3) by choosing first $M$, then $N$, sufficiently large, $T=M+N$. Now let $y(t)=x(t+T)$ and use (1.2) as above. These results

$$
y=\psi+e_{1} y+e_{2} y,
$$

where

$$
\psi(t)=r(t) x(T)+\int_{0}^{t} r(t-\tau)\left[f(\tau+T)-\int_{0}^{T} b(\tau+T, s) x(s) d s\right] d \tau
$$

$\mathcal{E}_{1} y(t)=-\int_{0}^{t} r(t-\tau) \int_{0}^{\tau} \beta(s+T) \alpha(\tau+T) A(\tau-s) y(s) d s d \tau$

$\varrho_{2} y(t)=\int_{0}^{t} r(t-\tau) \int_{0}^{\tau}[1-\gamma(s+T)] a(\tau-s) y(s) d s d \tau$.

Now

$$
\begin{aligned}
\int_{0}^{t} r(t-\tau) & \int_{0}^{T} b(\tau+T, s) x(s) d s d \tau \\
& =\int_{0}^{T} x(s)\left[\gamma(s) \psi_{1}(t, s)+\beta(s) \psi_{2}(t, s)\right] d s
\end{aligned}
$$

Here, by (1.3),

$$
\begin{aligned}
\psi_{1}(t, s) & =\int_{0}^{t} r(t-\tau) a(\tau+T-s) d \tau=\int_{T-s}^{t+T+s} r(t+T-s-\sigma) a(\sigma) d \sigma \\
& =-r^{\prime}(t+T-s)-\int_{0}^{T-s} r(t+T-s-\sigma) a(\sigma) d \sigma,
\end{aligned}
$$

so for $0 \leqslant s \leqslant T, M>0$,

Similarly,

$$
\begin{aligned}
\int_{0}^{M}\left|\psi_{1}(t, s)\right| d t & \leqslant\left\|r^{\prime}\right\|+\int_{0}^{T} a(\sigma) \int_{0}^{M+T}|r(t)| d t d \sigma \\
& \leqslant\left\|r^{\prime}\right\|+\|r\| \int_{0}^{T} a(\sigma) d \sigma \equiv K<\infty .
\end{aligned}
$$

$$
\begin{aligned}
\psi_{2}(t, s) & =\int_{0}^{t} r(t-\tau) \alpha(\tau+T) A(\tau+T-s) d \tau \\
\int_{0}^{\infty}\left|\psi_{2}(t, s)\right| d t & \leqslant\|r\| \int_{0}^{\infty} \alpha(\tau+T) A(\tau+T-s) d \tau \\
& \leqslant\|r\|\|\alpha A\|<\infty \quad(0 \leqslant s \leqslant T) .
\end{aligned}
$$


Since $\beta, \gamma \in L^{\infty}(0, T), x \in C[0, T]$, we deduce from (1.7), (1.10), (4.5), and (4.8) that $\psi \in L^{1}\left(\mathbf{R}^{+}\right)$.

From (4.6),

$$
\begin{aligned}
& \varrho_{1} y(t)=-\int_{0}^{t} y(s) \beta(s+T) \int_{s}^{t} r(t-\tau) \alpha(\tau+T) A(\tau-s) d \tau d s \\
& =-\int_{0}^{t} y(s) \beta(s+T) \int_{0}^{t-s} r(\sigma) \alpha(t-s+T+s-\sigma) A(t-s-\sigma) d \sigma d s .
\end{aligned}
$$

Thus

$$
\left\|\varrho_{1} z\right\| \leqslant\|z\|\|\beta\|_{\infty}\|r\|\|A(\cdot) \alpha(T+\cdot)\|
$$

Similarly, we see from (4.7) that

$$
\left\|\varrho_{2} z\right\| \leqslant\|z\|\|1-\gamma(T+\cdot)\|_{\infty}\left\|r^{\prime}\right\| \text {. }
$$

We use this together with (4.3), (4.4), (4.9) and the reasoning of the previous proof to see that $y \in L^{1}\left(\mathbf{R}^{+}\right)$with $\|y\| \leqslant 2\|\psi\|$. This proves Theorem 2.2.

We prove Theorem 3.2 under the assumptions of Theorem 2.1 with $p=1$ and $a \in L^{1}\left(\mathbf{R}^{+}\right)$; with obvious modifications, the same proof works if instead $b$ satisfies the hypotheses of Theorem 2.2.

Let $\varepsilon=1 / 2\|r\|$ and choose corresponding $T^{\prime}, c$, as for Theorem 2.1. Let $\eta=1 / 4\|r\|(\|a\|+\varepsilon)$ and choose $T>T^{\prime}$ so that $|g(x(t))| \leqslant \eta|x(t)|(t \geqslant T)$; this is possible, since $x(t) \rightarrow 0(t \rightarrow \infty)$ and $g(x)=o(x)(x \rightarrow 0)$. Now let $y(t)=x(t+T)$; as above, we obtain

$$
y=\varphi+\mathcal{L} y+\mathcal{G} y,
$$

where $\varphi \in L^{1}\left(\mathbf{R}^{+}\right)$, (4.2) holds with $p=1$, and

$$
\mathcal{G} z(t)=-\int_{0}^{t} r(t-\tau) \int_{0}^{\tau} b(\tau+T, s+T) g(z(s)) d s d \tau .
$$

Since $|b(\tau+T, s+T)| \leqslant|a(\tau-s)|+c(\tau-s)$, and since $|g(y(s))| \leqslant$ $\eta|y(s)|(s \geqslant 0)$

$$
|\mathfrak{G} y(t)| \leqslant \eta \int_{0}^{t}|r(t-\tau)| \int_{0}^{t}(|a(\tau-s)|+c(\tau-s))|y(s)| d s d \tau .
$$

Thus if we define $y_{\rho}$ as in the proof of Theorem 2.1, (4.10) holds with $y_{\rho}$ in place of $y$, and

$$
\left|y_{\rho}(t)\right| \leqslant|\varphi(t)|+\left|\mathcal{L} y_{\rho}(t)\right|+\left|\mathcal{G} y_{\rho}(t)\right| \quad(0 \leqslant t<\infty) .
$$

Our choice of $\eta$ implies that $\left\|\mathcal{G}_{\rho}\right\| \leqslant\left\|y_{\rho}\right\| / 4$; together with (4.2) $(p=1)$, this gives $\left\|y_{\rho}\right\| \leqslant 4\|\varphi\|(0<\rho<\infty)$. This proves Theorem 3.2.

V. Discussion and examples. Sufficient conditions for

$$
r, r^{\prime} \in L^{1}\left(\mathbf{R}^{1}\right)
$$

follow from a variant of the Wiener-Lévy theorem, proved by D. F. Shea and S. Wainger [8] and sharpened by G. S. Jordan and R. L. Wheeler [4]. 
According to [4, Theorem 1], (5.1) holds if

$$
\begin{gathered}
a=a_{1}+a_{2}, \quad a_{2} \in L^{1}\left(\mathbf{R}^{+}\right), \\
a_{1} \in L L^{1}\left(\mathbf{R}^{+}\right) \text {and is nonnegative, nonincreasing, } \\
\text { and convex on }(0, \infty),
\end{gathered}
$$

and

$$
\zeta+\hat{a}(\zeta) \neq 0 \quad(\operatorname{Re} \zeta \geqslant 0, \zeta \neq 0)
$$

where $\hat{a}$ is the Laplace transform of $a$, extended by continuity to $\{\operatorname{Re} \zeta=0$, $\zeta \neq 0\}$. (Condition (5.4) always holds when $a_{2} \equiv 0$, unless $a_{1}$ has a special piecewise linear form [3].)

Let

$$
b(t, s)=\alpha(t) \beta(s) A_{1}(t-s)+\gamma(s) A_{2}(t-s),
$$

with $\alpha, \beta, \gamma \in L^{\infty}\left(\mathbf{R}^{+}\right)$. (Kernels of this type have been analyzed by T. R. Kiffe [5] and J. J. Levin [6].) If $\gamma \equiv 1, \alpha(t) \rightarrow 1, \beta(t) \rightarrow 1(t \rightarrow \infty)$, the hypotheses of Theorem 2.1 hold if $a=A_{1}+A_{2}$ satisfies (5.2), (5.3), and (5.4) with $A_{1} \in L^{1}\left(\mathbf{R}^{+}\right)$and $A_{2} \in L^{p}\left(\mathbf{R}^{+}\right)$. For Theorem 2.2 it suffices to assume that $\alpha, \beta, \gamma \in L^{\infty}\left(\mathbf{R}^{+}\right), \gamma(\infty)=1, \alpha(t) \downarrow 1(t \uparrow \infty), A_{1} \geqslant 0, \alpha A_{1} \in L^{1}$, and that $a=A_{2}=a_{1}$ satisfies (5.3) and (5.4).

For Theorem 3.2, we must know in advance that $x$ exists on $\mathbf{R}^{+}$with $x(\infty)=0$. According to recent results of M. C. Smith [9], (see [5], [6] for earlier versions), this will be true if (with $k(x)=x+g(x)$ )

$$
\begin{aligned}
& f \in L^{1}\left(\mathbf{R}^{+}\right), k \in C(\mathbf{R}), x k(x)>0(x \neq 0), \\
& |k(x)| \leqslant M[1+K(x)] \text { and } K(x) \geqslant-M(x \in \mathbf{R}) \\
& \text { with } M<\infty, K(x) \rightarrow \infty(|x| \rightarrow \infty)
\end{aligned}
$$

(here $K(x)=\int_{0}^{x} k(y) d y$ ) and if $b$ and its derivatives satisfy certain sign and growth conditions. For the kernel $b$ of (5.5), either of the following sets of hypotheses ((5.7) or (5.8)), together with (5.6), is sufficient for Theorem 3.2:

$$
\begin{gathered}
A_{2} \equiv 0, A_{1}=a=a_{1} \in C^{1} \cap L^{1}(0, \infty) \text { and }(5.3) \text { holds, } \\
\alpha \in C^{1}\left(\mathbf{R}^{+}\right), \beta \in C\left(\mathbf{R}^{+}\right), \alpha(t) \downarrow 1,0 \leqslant \beta(t) \uparrow 1(t \uparrow \infty), \\
A_{1} \text { and } A_{2} \text { belong to } C^{1}(0, \infty) \text { and each satisfies }(5.3),
\end{gathered}
$$

$\beta$ and $\gamma$ are continuous and nondecreasing on $\mathbf{R}^{+}, \beta(\infty)=\gamma(\infty)=1$, (5.8ii)

$$
\alpha \in C^{1}\left(\mathbf{R}^{+}\right), \alpha(t) \downarrow 0(t \uparrow \infty), \alpha A_{1} \in L^{1}\left(\mathbf{R}^{+}\right) .
$$

Another kernel for which our results hold (and which was studied in [6]) is

$$
b(t, s)=a_{1}(\alpha(t)(t-s))
$$

where $a=a_{1} \in L^{1}\left(\mathbf{R}^{+}\right)$, (5.3) and (5.4) hold, $\alpha \in C\left(\mathbf{R}^{+}\right)$, and $\alpha(t) \downarrow 1$ $(t \uparrow \infty)$. Then by the mean value theorem, 


$$
\begin{aligned}
0 & \leqslant a_{1}(t-s)-b(t+T, s+T) \\
& \leqslant-[\alpha(T)-1](t-s) a_{1}^{\prime}(t-s) \equiv c_{T}(t-s)
\end{aligned}
$$

and $\left\|c_{T}\right\| \rightarrow 0$ as $T \rightarrow \infty$. Thus $b$ satisfies the hypotheses of Theorem 2.1 with $p=1$. For Theorem 3.2, certain additional assumptions are again needed to ensure that a solution $x$ exists with $x(\infty)=0$. See [6], [9].

For an example where $x \notin L^{1}\left(\mathbf{R}^{+}\right)$but $x(t) \rightarrow 0$ as $t \rightarrow \infty$, choose $a(t)=$ $e^{-t} / 4$. Then (1.3) reduces to an ordinary differential equation, and $r(t)=e^{-t / 2}\left(1+\frac{1}{2} t\right)$. Let $A=a_{1}$ satisfy (5.3) with $A(\infty)=0$ and $\int_{0}^{\infty} A(t) d t$ $=\infty$. Note that for $t \geqslant 1$,

$$
q(t) \equiv \int_{0}^{t} r(s) A(t-s) d s \geqslant \int_{0}^{1} r(s) A(t-s) d s>2 A(t) / 3 .
$$

Thus $q>0$ and $q \notin L^{1}\left(\mathbf{R}^{+}\right)$, but $q(\infty)=0$, since $r \in L^{1}$ and $A(\infty)=0$. Now let

$$
\begin{aligned}
\beta(s) & =\beta_{0} \quad(0 \leqslant s \leqslant 1) \\
& =0 \quad(1<s<\infty),
\end{aligned}
$$

where the positive number $\beta_{0}$ is chosen so that

$$
\int_{0}^{1}\left[a(t)+\beta_{0} A(t)\right] d t<\frac{1}{2} .
$$

Let $b(t, s)=a(t-s)+\beta(s) A(t-s), f \equiv 0$, and let $x$ be the solution of (1.1). Clearly $\frac{1}{2} \leqslant x(t) \leqslant 1(0 \leqslant t \leqslant 1)$, and the change of variables $y(t)=$ $x(t+1)$ gives

$$
y^{\prime}(t)+\int_{0}^{t} a(t-s) y(s) d s=-\int_{0}^{1}\left[a(t-s)+\beta_{0} A(t-s)\right] x(s) d s
$$

$(t \geqslant 0), y(0)=x(1)$. Thus by (1.2),

$$
\begin{aligned}
y(t)= & r(t) x(1)-\int_{0}^{1} x(s) \int_{0}^{t} r(t-\tau) a(\tau+1-s) d \tau d s \\
& -\beta_{0} \int_{0}^{1} x(s) \int_{0}^{t} r(t-\tau) A(\tau+1-s) d \tau d s \\
\equiv & r(t) x(1)+y_{1}(t)+y_{2}(t) .
\end{aligned}
$$

But, as in the proof of Theorem 2.2,

$$
\begin{aligned}
\int_{0}^{t} r(t & -\tau) a(\tau+1-s) d \tau \\
& =-r^{\prime}(t+1-s)-\int_{0}^{1-s} r(t+1-s-\sigma) a(\sigma) d \sigma
\end{aligned}
$$

so $y_{1} \in L^{1}\left(\mathbf{R}^{+}\right)$and $y_{1}(t) \rightarrow 0(t \rightarrow \infty)$. On the other hand, 


$$
\begin{aligned}
\int_{0}^{t} r(t-\tau) A & (\tau+1-s) d \tau \\
= & q(t+1-s)-\int_{0}^{1-s} r(t+1-s-\sigma) A(\sigma) d \sigma \\
= & q(t+1-s)+O\left(t e^{-t}\right)
\end{aligned}
$$

as $t \rightarrow \infty$, uniformly in $0 \leqslant s \leqslant 1$. Therefore $x(t) \rightarrow 0(t \rightarrow \infty)$ but $x \notin$ $L^{1}\left(\mathbf{R}^{+}\right)$, as claimed.

\section{REFERENCES}

1. S. I. Grossman and R. K. Miller, Perturbation theory for Volterra integrodifferential systems, J. Differential Equations 8 (1970), 457-474.

2. __ Nonlinear Volterra integrodifferential systems with $L^{1}$ kernels, J. Differential Equations 13 (1973), 551-566.

3. K. B. Hannsgen, Indirect abelian theorems and a linear Volterra equation, Trans. Amer. Math. Soc. 142 (1969), 539-555.

4. G. S. Jordan and R. L. Wheeler, A generalization of the Wiener-Lévy theorem applicable to some Volterra equations, Proc. Amer. Math. Soc. 57 (1976), 109-114.

5. T. R. Kiffe, On nonlinear Volterra equations of nonconvolution type, J. Differential Equations 22 (1976), 349-367.

6. J. J. Levin, A nonlinear Volterra equation not of convolution type, J. Differential Equations 4 (1968), 176-186.

7. R. K. Miller, Asymptotic stability properties of a linear Volterra integrodifferential equation, J. Differential Equations 10 (1971), 485-506.

8. D. F. Shea and S. Wainger, Variants of the Wiener Lévy theorem, with applications to stability problems for some Volterra integral equations, Amer. J. Math. 97 (1975), 312-343.

9. M. C. Smith, A nonlinear Volterra equation of nonconvolution type, Thesis, Virginia Polytechnic Institute and State Univ., Blacksburg, Virginia, 1977.

Department of Mathematics, Virginia Polytechnic Institute and State University, BLACKSBURG, ViRgINIA 24061 